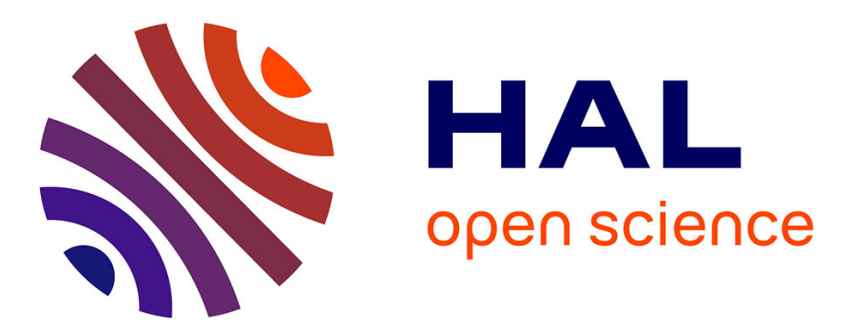

\title{
Clinical efficiency of a natural resin fluoride varnish (Shellac F) in reducing dentin hypersensitivity
}

\author{
B.-T. Hoang-Dao, H. Hoang-Tu, N.-N. Tran-Thi, G. Koubi, J. Camps, I.
}

About

\section{- To cite this version:}

B.-T. Hoang-Dao, H. Hoang-Tu, N.-N. Tran-Thi, G. Koubi, J. Camps, et al.. Clinical efficiency of a natural resin fluoride varnish (Shellac F) in reducing dentin hypersensitivity. Journal of Oral Rehabilitation, 2009, 36 (2), pp.124-131. 10.1111/j.1365-2842.2008.01907.x . hal-03552172

\section{HAL Id: hal-03552172 \\ https://hal.science/hal-03552172}

Submitted on 2 Feb 2022

HAL is a multi-disciplinary open access archive for the deposit and dissemination of scientific research documents, whether they are published or not. The documents may come from teaching and research institutions in France or abroad, or from public or private research centers.
L'archive ouverte pluridisciplinaire HAL, est destinée au dépôt et à la diffusion de documents scientifiques de niveau recherche, publiés ou non, émanant des établissements d'enseignement et de recherche français ou étrangers, des laboratoires publics ou privés. 


\title{
Clinical efficiency of a natural resin fluoride varnish (Shellac F) in reducing dentin hypersensitivity
}

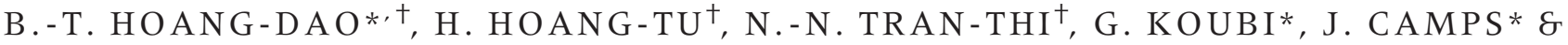 \\ I. ABOUT* *Laboratoire IMEB, Faculté d'Odontologie, Université de la Méditerranée, Marseille, France and ${ }^{\dagger}$ Faculty of Odonto- \\ Stomatology, University of Medicine and Pharmacy, HCM City, Viet Nam
}

\begin{abstract}
SUMMARY Previous in vitro permeability and scanning electron microscopic studies have demonstrated the effectiveness of a new natural based-resin varnish (Shellac F) in dentin permeability reduction and effective tubule occlusion. The aim of this randomized double-blind, controlled, split mouth 8-week clinical study was to evaluate the efficiency of Shellac $F$ in reducing dentin hypersensitivity. Ten patients (eight women: two men) completed the study. A quadrant including at least one hypersensitive tooth (Visual Analog Scale - VAS $=15 \mathrm{~mm}$ to air blast) was considered as a unit and randomly assigned to different groups for Shellac F, Duraphat ${ }^{\circledR}$, Isodan $^{\circledR}$. Three applications of each material were completed at days 0,1 and 7. The subjective response was assessed by tactile and thermal/evaporative methods. Data were collected at baseline and after the first application, at $15 \mathrm{~min}, 1,7,14,28$ and 56 days.
\end{abstract}

Analysis was based on Kruskall-Wallis test, Wilcoxon signed rank test and the method of the least square means. No statistically significant difference was noted between Shellac $F$ and the two control materials. Regardless of the type of stimulus, Shellac F showed significant immediate and progressive continuous efficiency in reducing dentin hypersensitivity until 56 days (VAS of $14 \pm 12 \mathrm{~mm}$ and provoking pain force of $89 \pm 12 \mathrm{cN}$, respectively, compared with $38 \pm 23 \mathrm{~mm}$ and $41 \pm 10 \mathrm{cN}$ at baseline), corresponding to a highly effective relief dentin hypersensitivity. Shellac F reduced dentin hypersensitivity and did not differ from the two desensitizing agents used as controls.

KEYWORDS: Shellac F, natural resin-based fluoride varnish, desensitizingagent, dentinhypersensitivity

\section{Introduction}

Dentin hypersensitivity $(\mathrm{DH})$ is characterized by a short, sharp pain arising from exposed dentin in response to stimuli, typically thermal, evaporative, tactile, osmotic or chemical, which cannot be ascribed to any other dental defect or disease (1-4). This discomfort is a common finding in adult populations (5). The prevalence of cervical DH varies depending on the studied populations and methods used (6). The increase of longevity of dentition through periodontal therapy and plaque control procedures may increase the incidence of dentin hypersensitivity. The ultimate goal in the treatment of $\mathrm{DH}$ is the immediate and the permanent pain relief (5). Some recent reviews on the management of DH provided sufficient information on the efficiency of the therapeutic products used (7-9). Currently, there are two main approaches for the treatment of DH, namely tubular occlusion and blocking nerve activity through the inhibition of direct ionic diffusion (10).

Depending on the perceived severity of the condition and the number of teeth involved, active management of DH can be performed separately or in combination using over-the-counter (OTC) and in-office therapies. OTC desensitizing agents such as a desensitizing dentifrice, used by patients, generally are simple to administer. Dental professionals can deliver a wider range of more complex and more potent desensitizing treatment. Numerous agents have been tested in vitro 
and in clinical studies and showed their DH relief effects, including fluoride, potassium nitrate, oxalate and calcium phosphates (11). Fluorides such as sodium fluoride and stannous fluoride can reduce dentin sensitivity (12). Fluorides decrease the permeability of dentin in vitro (13), possibly by precipitation of insoluble calcium fluoride within the tubules (11). Fluoride varnishes are recognized by the Federal Drug Administration as a device to be used as a desensitizing agent and a cavity lining varnish $(14,15)$.

Shellac F is an experimental fluoride varnish, which was fabricated in 1997 by the Faculty of OdontoStomatology, University of Medicine and Pharmacy of Ho Chi Minh City, in a project of Ministry of Health of Viet Nam. It is composed of $5 \% \mathrm{NaF}$ in shellac, a natural polymer secreted by an insect named Laccifer lacca kerr, which lives on some specific trees in South East Asian countries such as China, India, Thailand and Viet Nam. This resin has been largely used in the traditional procedure of tooth lac, a popular custom of many Pacific countries in previous centuries. A protective effect for the dentition including dentin hypersensitivity management has been reported throughout the period of use. This observation has been also verified by some clinical studies in the past few decades. Recently, the adequate cellular compatibility and the efficiency of Shellac F at reducing human dentin hydraulic conductance have been demonstrated in an in vitro study (16). These results have supported the potential of a clinical application of Shellac F as a desensitizing agent. The objective of this clinical study was to evaluate the efficiency of the Shellac F in reducing DH in human dentition.

\section{Materials and Methods}

This double-blind, controlled, split-mouth designed clinical study was conducted to evaluate the desensitizing efficiency of a new fluoride varnish, Shellac $\mathrm{F}^{*}$ in subjects with cervical DH. Two other commercially available products: a fluoride varnish: Duraphat ${ }^{\circledR+}$ and a fluoride containing desensitizing agent: $\operatorname{Isodan}^{\circledR \ddagger}$ were used as controls (Table 1). For ethical reasons, we did not include a placebo group in the study.
The experimental design followed the Guidelines for the design and conduct of clinical studies on dentin hypersensitivity of Holland et al. (4). Subjects were recruited from patients presenting cervical erosion lesions who came to the Consultation Unit of the Faculty of Odonto-Stomatology, University of Medicine and Pharmacy, HCM City, Viet Nam. The inclusion and exclusion criteria are showed in Table 2. A differential diagnosis between $\mathrm{DH}$, root dentin sensitivity and pulpal pathology was performed (17).

Patients provided signed written informed consent prior to study enrolment. To minimize the bias, patients

Table 1. Composition of three materials used in the study

\begin{tabular}{ll}
\hline Material & Components \\
\hline Shellac F & $\begin{array}{c}\text { Sodium fluoride }(5 \%) \text {, shellac, modified epoxy } \\
\text { resin, acetone, silica } \\
\text { Duraphat }{ }^{\circledR}\end{array}$ \\
$\begin{array}{c}\text { Sodium fluoride }(5 \%), \text { ethanol, white bee wax } \\
(\text { E901), gum lac (E904), colophane, mastic, }\end{array}$ \\
$\begin{array}{l}\text { saccharine (E954), frasberry essence } \\
\text { Potassium nitrate, sodium fluoride, HEMA, } \\
\text { Isodan }{ }^{\circledR}\end{array}$ \\
\hline
\end{tabular}

Table 2. Inclusion and exclusion criteria

Inclusion criteria

Have at least two different quadrants which have sensitive teeth with sound exposed cervical dentin on the facial surface showing a response of $\geq 15 \mathrm{~mm}$ on a 100 -mm Visual Analog Scale (VAS) to a 1 -s evaporative stimulus.

Exclusion criteria

Exclusion criteria for subject

Current and/or previous use of professional desensitizing treatment

Use of over-the-counter desensitizing products within the previous 6 weeks

Long-term use anti-inflammatory, analgesic and psychotropic drugs

Pregnancy or breast feeding

Allergies and idiosyncratic responses to product ingredients

Eating disorders

Systemic conditions that cause or predispose patients to develop dentin hypersensitivity

(for example, chronic acid regurgitation)

Excessive dietary or environmental exposure to acids

Periodontal surgery in the preceding 3 months

Orthodontic appliance treatment within previous 3 months

Exclusion criteria for teeth

Carious and/or restored tooth

Tooth relates with any kind of prothese

Teeth or supporting structures with any other

painful pathology or defects 
were provided with identical toothbrushes, dentifrices and oral hygiene instructions. Different quadrants of each patient were considered as a unit and randomly assigned to different test groups in according to this rule:

1 Patient with two sensitive quadrants: a quadrant for Shellac F, and the other for one of the two controls.

2 Patient with three sensitive quadrants: a quadrant for each of the three materials.

3 Patient with four sensitive quadrants: two quadrants for Shellac F, and two others for the two controls.

Ten subjects (eight women and two men), ranging from 22 to 52 years of age were selected, with a total of 40 test teeth. The distribution of the materials in the quadrants allowed a total number of 60 paired comparisons (Table 3 ). This was obtained by putting each paired products in different quadrants of the same patient.

One trained dentist examiner (Tran-Thi NN) was responsible for applying the stimuli and enrolment of the subjects into the study. A second trained examiner (Hoang-Dao BT) was responsible for applying the materials and a third dental examiner (Hoang-Tu $\mathrm{H}$ ) was in charge of collecting subjects' responses during the recall visits. Tactile and thermal/evaporative test were used, respectively, to assess dentin hypersensitivity at baseline and postoperation.

The tactile threshold was first measured by using a scratchometer calibrated in centi-Newton $(0-100 \mathrm{cN})$ (18) as it is generally accepted that tactile stimuli are

Table 3. Distribution of treated teeth according to the material used and the resulting total paired comparisons

\begin{tabular}{|c|c|c|c|c|}
\hline \multirow[b]{2}{*}{$\begin{array}{l}\text { Patients with two } \\
\text { test quadrants }\end{array}$} & \multirow{2}{*}{$\begin{array}{l}\text { Material } \\
\text { Shellac F } \\
\text { Duraphat }{ }^{\circledR} \\
\text { Isodan }\end{array}$} & \multicolumn{2}{|c|}{$\begin{array}{l}\text { Total no. } \\
\text { treated } \\
\text { teeth }\end{array}$} & \multirow{2}{*}{$\begin{array}{l}\begin{array}{l}\text { No. paired } \\
\text { comparisons }\end{array} \\
5\end{array}$} \\
\hline & & $\begin{array}{l}4 \\
3 \\
2\end{array}$ & 9 & \\
\hline $\begin{array}{l}\text { Patients with three } \\
\text { test quadrants }\end{array}$ & $\begin{array}{l}\text { Shellac F } \\
\text { Duraphat }{ }^{\circledR} \\
\text { Isodan }\end{array}$ & $\begin{array}{r}10 \\
6 \\
5\end{array}$ & 21 & 28 \\
\hline $\begin{array}{l}\text { Patient with four } \\
\text { test quadrants }\end{array}$ & $\begin{array}{l}\text { Shellac F } \\
\text { Duraphat }^{\circledR} \\
\text { Isodan }\end{array}$ & $\begin{array}{l}6 \\
1 \\
3\end{array}$ & 10 & 27 \\
\hline Total & & & 40 & 60 \\
\hline
\end{tabular}

Each paired comparison was obtained after putting each paired products in two different quadrants of the same patient. less invasive than thermal stimuli (19). The compressive force was applied perpendicular to the cervical surface of the tooth. The examiner moved the tip of the probe across the exposed dentin surface in a systemic manner in a horizontal direction to locate the stimulated area. The indicator needle determined the applied force, which started from 0 and increased until the upward transition (initial positive response) was reached. The maximal applicable force was $100 \mathrm{cN}$.

Following tactile stimulation, the subject's response to thermal/evaporative stimulus was assessed using a 1-s blast of cold air delivered from a dental air syringe (40-65 p.s.i., $22 \pm 2{ }^{\circ} \mathrm{C}$ ) applied $1 \mathrm{~cm}$ away from and perpendicular to the test area. Adjacent teeth were isolated during evaluation with cotton rolls. Each patient was asked to indicate the pain intensity in a 100-mm VAS (Visual Analog Scale) labeled from 'no pain' (0 mm) to 'extreme pain' (100 mm).

For both types of stimulus, the measurement was performed in triplicate with an interval of $5 \mathrm{~min}$ between stimulations. Triple measurements were made to ensure that the pain was reproducible. The time elapsed between each measurement was much longer than the refractory period of sensory nerves (20). Mean values were collected for statistical analysis. Treatment application was initiated at three time periods (days 0,1 and 7) for all of the three materials. The teeth were cleaned with water spray and gently dried with air blast. The isolation was obtained using cotton rolls together with the aid of a high potency saliva ejector. Shellac F was applied with a thick coat over the exposed cervical area by an applicator tip followed by an adequate air dry $\left(40-65\right.$ p.s.i., $22 \pm 2{ }^{\circ} \mathrm{C}, 10 \mathrm{~s}, 1 \mathrm{~cm}$ distance). The two control materials were applied according to the manufacturers' instructions. Product application was similar to that of Shellac F. The whole process took $10 \mathrm{~min}$. It was the same in all patients and for all materials. The subjects were instructed to refrain from brushing, flossing and eating for at least two hours after each application to avoid removing the varnish mechanically.

The efficiency of the treatments was assessed at six examination periods: 15 min after the first application (postoperative), then at days 1, 7, 14, 28 and 56, respectively, by tactile and thermal/evaporative stimuli. The statistical analysis was performed in four steps for both clinical parameters (tactile stimulus and thermal/evaporative stimulus). First, to know whether the hypersensitive teeth were homogeneously distributed 
among the three groups a nonparametric KruskallWallis test was performed on the baseline values. Second, to compare the baseline level and the $15 \mathrm{~min}$ postoperative results to see whether the treatments had immediate efficiency at reducing $\mathrm{DH}$, a nonparametric Wilcoxon signed rank test was performed. Third, to pinpoint the differences among the three materials, a nonparametric Kruskall-Wallis test was performed. Finally, to evaluate the DH decrease over time, a regression analysis, by the method of the least squares was performed. The significance level was set at $5 \%$ for all statistical analyses.

\section{Results}

Recruitment to DH studies based on the Holland et al. (4) recommendations was difficult and published studies generally report small numbers of participants per group. In this work, 13 subjects were recruited but only 10 of them completed the study. However, sufficient teeth were treated to enable 60 paired comparisons between different quadrants in the same patient (Table 3).

First, the baselines values of the three groups, recorded before the first application at day 0 , were not statistically different according to the KruskallWallis test (Figs 1 and 2). This means that the hypersensitive teeth were equally distributed in the three groups receiving the three materials.

Second, the Wilcoxon signed rank test showed a statistically significant difference between the baseline and the 15 min postoperative values indicating that the

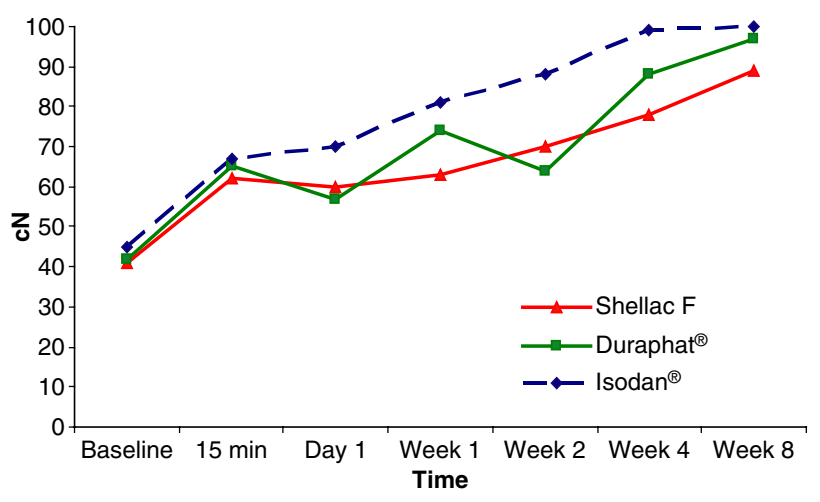

Fig. 1. Reduction of dentin hypersensitivity (DH) over time (tactile threshold $\mathrm{cN}$ ). All materials induced a significant decrease of dentin hypersensitivity symptom showed by a statistical increase of tactile threshold. No statistically significant difference was found among the three materials.

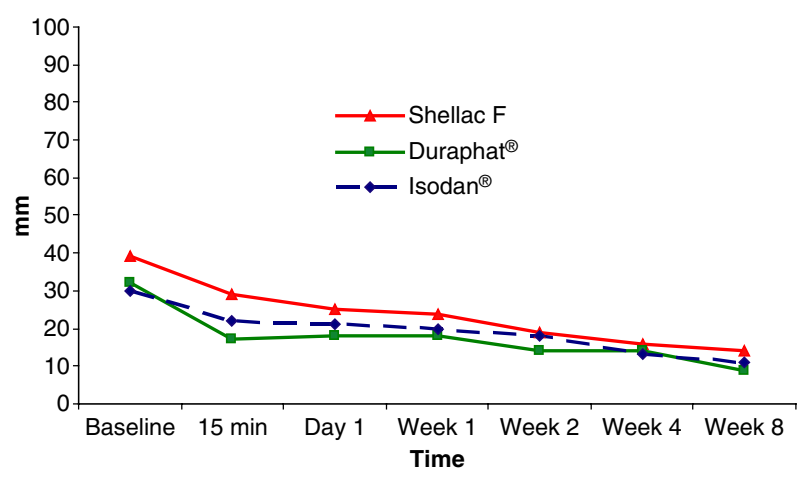

Fig. 2. Reduction of dentin hypersensitivity (DH) over time (sensitivity to air-blasting Visual Analog Scale - VAS). All materials induced a significant decrease of dentin hypersensitivity symptom showed by a decrease of 100-mm VAS indicator. No statistically significant difference was found among the three materials.

three materials had an immediate efficiency at reducing DH. The tactile threshold significantly increased from $42 \pm 13$ to $65 \pm 24 \mathrm{cN}$ for Duraphat ${ }^{\circledR}(P<0.001)$, from $45 \pm 20$ to $67 \pm 30 \mathrm{cN}$ for Isodan $^{\circledR}(P<0.001)$ and from $41 \pm 10$ to $62 \pm 20 \mathrm{cN}$ for Shellac $\mathrm{F}(P<0 \cdot 001$; Fig. 1). The VAS significantly decreased from $32 \pm 16$ to $17 \pm 12 \mathrm{~mm}(P<0.001)$ for Duraphat ${ }^{\circledR}$, from $30 \pm 18$ to $22 \pm 19 \mathrm{~mm}(P<0.001)$ for Isodan $^{\circledR}$ and from $39 \pm 22$ to $29 \pm 19 \mathrm{~mm}$ for Shellac $\mathrm{F}(P=0.001)$ (Fig. 2).

Third, the Kruskall-Wallis tests did not show any statistically significant difference among the three materials for each period of time, neither for tactile stimulus (Fig. 1) nor for thermal/evaporative stimulus (Fig. 2) (ns). Fourth, the regression analysis showed a statistically significant linear correlation between the $15 \mathrm{~min}$, and 1, 7, 14, 28 and 56-day postoperative values for each material. The recorded tactile threshold $(\mathrm{cN})$ increased linearly for Duraphat ${ }^{\circledR} \quad(P=0.02$; $y=0.53 x+63.5$ with $\left.R^{2}=0.8\right), \quad$ Isodan $^{\circledR} \quad(P=0.03$; $y=0.47 x+75$ with $\left.R^{2}=0.7\right)$ and Shellac F $(P=0.02$; $y=0 \cdot 4 x+61 \cdot 8$ with $R^{2}=0 \cdot 95$; Fig. 1 ). This means that sensitivity threshold to tactile stimulus increased regularly for all of three materials, during 56 days after the first material application (49 days after the whole treatment). The recorded VAS ( $\mathrm{mm}$ ) decreased linearly for Duraphat ${ }^{\circledR} \quad(P=0 \cdot 02 ; \quad y=-0 \cdot 11 x+18 \quad$ with $\left.R^{2}=0 \cdot 8\right), \quad$ Isodan $^{\circledR} \quad(P=0 \cdot 02 ; \quad y=-0 \cdot 17 x+21 \quad$ with $\left.R^{2}=0 \cdot 8\right)$ and Shellac F $(P=0 \cdot 04 ; y=-0 \cdot 14 x+24 \cdot 8$ with $R^{2}=0 \cdot 6$; Fig. 2 ). The continuous decrease in VAS $(\mathrm{mm})$ over time during 56 days confirms that the 
treatment protocol applied in this study is sufficient to relieve pain during this period of time, for all of three materials.

\section{Discussion}

Cervical DH is a significant clinical problem in dentistry because it affects a large percentage of the population, and as such there is no completely effective, conservative and permanent treatment (6). On the basis of the hydrodynamic mechanism of Brännström (21), dentin hypersensitivity treatments are reliant on dentin permeability reduction by using potential intratubular occluding agents. Clinicians have attempted several strategies to treat this discomfort, including topical fluorides, which have been used for at least 60 years to treat DH $(22,23)$. On the basis of previous in vitro evidence, topical fluorides appear to create a barrier by precipitating $\mathrm{CaF}_{2}$ at the exposed dentin and, consequently, decrease DH (24). The natural resins contained in fluorides varnishes may provide an additional barrier effect, although this has not been experimentally tested (6).

Shellac $\mathrm{F}$ is a new natural resin-based varnish containing 5\% sodium fluoride. A recent in vitro study showed that Shellac $\mathrm{F}$ had a significant efficiency in reducing dentin permeability and presented a capacity to form a crystalline layer with dentinal tubular plugs (16). This permeability reduction was comparable to control materials which contain sodium fluoride and have been investigated in other studies (25).

This study was conducted to clinically evaluate the efficiency of Shellac F as a desensitizing agent. Duraphat ${ }^{\circledR}$ and Isodan ${ }^{\circledR}$ were used as positive controls. These two materials are commercially available and contain sodium fluoride in their composition. Duraphat ${ }^{\circledR}$ is a yellow fluoride varnish with the base nature closely like Shellac F (Table 1). By contrast, Isodan ${ }^{\circledR}$ is presented as a transparent fluid gel and contains HEMA. The treatment protocol applied in this study followed the manufacturer's instruction of these materials for dentin hypersensitivity management. The efficiency of Isodan ${ }^{\circledR}$ in reducing dentin permeability $(78 \pm 5 \%)$ was reported by Camps et al. (25) and numerous studies advocated the clinical effects of sodium fluoride-contain products $(26,27)$. In a 30 -day clinical study, Corona et al. used Duraphat ${ }^{\circledR}$ as a positive control to evaluate the efficiency of low-level galium-aluminium-arsenide (GaAlAs) laser. Both treatments showed similar overall performance and provided a gradual decrease in dentin hypersensitivity (26). Ritter et al. (28) in a 24 week study also reported the efficiency of Duraphat ${ }^{\circledR}$, which was used as a positive control for testing another fluoride varnish.

With regard to the other criteria followed in the protocol, the split mouth study design was chosen in this work as it is a highly effective and efficient model for professional application of sensitivity products. The subject acts as his own control which is very powerful tool statistically, and the methodology of choice (29). Fully replicated randomized blocks with several observations of each treatment in each block, as performed here, improves the statistical analysis power (30). No difference among the three materials was reported here. A large sample size associated with a small standard deviation is always desirable and increases the power (beta risk) of a statistical analysis. The VAS data reported here present a large standard deviation that may raise question about the outcome of the study. However, two specific points are in favour of the validity of this work. The first point is the special design of the study, which permitted us to make 60 valid paired-comparisons with only 10 patients. The special care taken to recruit the patients limited their number but working on several teeth within different quadrants on the same patient increased the number of valid paired-comparisons and limited the unavoidable variations among patients when recording pain. The second point is in favour of the use of VAS. This measurement scale has already been used in dentistry. It was compared with other scales and can be considered as a valid tool to assess dental pain (31). In addition, the statistical analysis of this work failed to show a significant difference among the materials not only when performed on the VAS data but also when using the scratchometer values, which presented a much lower standard deviation. As both parameters varied accordingly and indicated a strong decrease of DH over time, on may consider the low power of the statistical analysis performed with the VAS values was counterbalanced and confirmed by the high power of the statistical analysis performed on the scratchometer data.

Pain assessment is always a perplexing challenge. Regardless of the method and materials employed, the evaluation of treatments for $\mathrm{DH}$ is not simple (26). There is no doubt that patient's self-report discomfort arising from various stimuli, but the highly subjective nature of the condition makes it extremely difficult to 
evaluate DH objectively. This is particularly true when evaluating the efficiency of desensitizing agents in the clinical study setting (32). Pain associated with DH is difficult to quantify and reproduce, this explains the large standard deviation reported here when measuring pain with the VAS (33). It can be evaluated by stimulus and response-based assessments (34). The stimulusbased method involves the measurement of a pain threshold, and the response-based method involves the estimation of pain severity. Many types of stimuli can cause $\mathrm{DH}$, but some of these stimuli are impractical to use in qualifying or quantifying $\mathrm{DH}$ in the clinical environment (34). The tactile, evaporative and cold test is recommended because they are physiological and controllable (34). At least two types of stimulus should be applied and the less invasive should be performed first (32). In this work, we used the tactile test as stimulus-based assessment and the thermal/evaporative test as response-based assessment.

The scratchometer was used to determine the pain threshold of patients to tactile stimulus and was applied first. As scratching was completed prior to air blast, one may suppose that the scratching test did not damage the dentin surface to such an extent that it increased sensitivity to air blast (19). A previous study showed that the dentinal grooves made by a probe under clinically relevant forces were between 20 and $30 \mu \mathrm{m}$ wide (19). The dentin area exposed by scratching was so small compared with the dentin area exposed to air blast that it likely did not influence the prevalence of sensitivity to air blast (19). The scratchometer permits obtaining a quantitative and objective assessment because the initial pain threshold is indicated just at the moment when the pain starts without any other subjective interpretation.

The VAS was used to assess the subjective perception of the patients with thermal/evaporative stimuli. The subject response was quantified by using the VAS, which is considered as preferable to a numerical rating scale where the subject rates pain intensity on a 100$\mathrm{mm}$ scale comprising four distinct categories. The VAS offers the advantage of being a continuous scale, thus providing quantitative measurements that are readily averaged and tested with parametric statistics (34). This scale has been reported as reliable in the literature for pain assessment $(35,36)$ and can complement the tactile testing method which has been reported somewhat uncertain with resin-containing materials (17). Dentin hypersensitivity was assessed recording the response to mechanical and evaporative stimuli in triplicate. The time elapsed between the measurements ( $5 \mathrm{~min}$ ) was much longer than the refractory period of sensory nerves (20). In addition, the air blast was very short (1-s) (37) and dentin may reabsorb water from saliva, which has been shown to be maximal in dehydrated dentin during the early time periods (38).

The results showed that Shellac F had an immediate effect in reducing hypersensitivity and this effect persisted throughout the study, regardless of the stimulus. Hypersensitivity means for both stimuli decreased continually throughout the study with the most significant effect observed at the end (at day 56). This effect was similar to that of two control materials which were previously tested $(6,25-27)$ and currently used in clinical practice. These results also supported the results of the previous in vitro study in which all of these three materials showed a significant and gradual dentin permeability decrease (16). A placebo group might be helpful to determine more clearly if any of the results obtained were due to a placebo effect and give a more interesting interpretation of the results. However, a relative long-term follow-up, such as the one reported here, might allow us to record the expression of the maximum efficiency of the 'active' agent, whilst minimizing the magnitude of the 'placebo effects' (4).

Dentin hypersensitivity reduction as observed in this study is somewhat surprising, because the $\mathrm{CaF}_{2}$ precipitates formed on the outer dentin was expected to have been washed away by the saliva and toothbrush abrasion, reopening the dentinal tubules and triggering hypersensitivity. However, it has been shown that after topical application of $5 \% \mathrm{NaF}$, most of the fluoride was released within 2 weeks, with only small amounts of fluoride being released up to 21 weeks after treatment (39). In this study, the data showed a significant reduction of DH immediately after the first application and throughout 8 weeks, regardless of the stimulus. This result corroborates the results of other studies (27) and suggests an application of Shellac $\mathrm{F}$ as a desensitizing agent. The resinous material of Shellac F may act as a barrier, preventing sodium fluoride wash out.

This work adhered to the recommendation of conducting DH studies (4). Ten patients followed the complete protocol, giving 40 teeth for the statistical analysis. The limited sample size is commonly found in DH clinical studies $(40,41)$, which requires a highly strict protocol to evaluate such a subjective and multifactor condition in human. The unequilibration of 
gender was also present in this study because females appear to suffer more than males due to their overall heath care and oral hygiene awareness (42). During the study, subject response to 'everyday' stimuli was also undertaken to assess the individual's overall sensitivity but the statistical analyses were not engaged because of the variety according to multi-factors as teeth, aetiology, stimuli, individual habits and responses. Within the limitations of this study, it was demonstrated that Shellac F had significant effects in reducing DH with both tactile and thermal/evaporative stimuli. This hypersensitivity reduction appears immediately after the first application and persisted throughout the duration of study, until 8 weeks.

\section{Acknowledgments}

This work was undertaken at the Faculty of OdontoStomatology, University of Medicine and Pharmacy, HCM city, Viet Nam and was supported by institutional funding from French "Ministère de l'éducation nationale, de l'enseignement supérieur et de la recherche" and the Ministry of Education and Formation of Viet Nam.

\section{References}

1. Addy M. Clinical aspects of dentine hypersensitivity. Proc Finn Dent Soc. 1992;88(Suppl. 1):23-30.

2. Orchardson R, Collins WJ. Clinical features of hypersensitive teeth. Br Dent J. 1987;162:253-256.

3. Canadian Advisory Board on Dentin Hypersensitivity. Consensus-based recommendations for the diagnosis and management of dentin hypersensitivity. J Can Dent Assoc. 2003;69:221-226.

4. Holland GR, Narhi MN, Addy M, Gangarosa L, Orchardson R. Guidelines for the design and conduct of clinical trials on dentine hypersensitivity. J Clin Periodontol. 1997;24:808813.

5. Dababneh RH, Khouri AT, Addy M. Dentine hypersensitivity - an enigma? A review of terminology, mechanisms, aetiology and management. Br Dent J. 1999;187:606-61 1; Discussion 603.

6. Fischer C, Fischer RG, Wennberg A. Prevalence and distribution of cervical dentine hypersensitivity in a population in Rio de Janeiro, Brazil. J Dent. 1992;20:272-276.

7. Chabanski MB, Gillam DG. Aetiology, prevalence and clinical features of cervical dentine sensitivity. J Oral Rehabil. 1997;24:15-19.

8. Drisko C. Dentine hypersensitivity - dental hygiene and periodontal considerations. Int Dent J. 2002;52:385-393.

9. Pashley DH. Potential treatment modalities for dentine hypersensitivity: in-office products. In: Addy M, Embery G,
Edgar WM, Orchardson R, eds. Tooth wear and sensitivity. London (UK): Martin Dunitz, 2000:351-365.

10. Gangarosa LP Sr. Current strategies for dentist-applied treatment in the management of hypersensitive dentine. Arch Oral Biol. 1994;39(Suppl.):101S-106S.

11. Orchardson R, Gillam DG. Managing dentin hypersensitivity. J Am Dent Assoc. 2006;137:990-998; Quiz 1028-9.

12. Morris MF, Davis RD, Richardson BW. Clinical efficacy of two dentin desensitizing agents. Am J Dent. 1999;12:72-76.

13. Greenhill JD, Pashley DH. The effects of desensitizing agents on the hydraulic conductance of human dentin in vitro. J Dent Res. 1981;60:686-698.

14. Bawden JW. Fluoride varnish: a useful new tool for public health dentistry. J Public Health Dent. 1998;58:266-269.

15. Beltran-Aguilar ED, Goldstein JW, Lockwood SA. Fluoride varnishes. A review of their clinical use, cariostatic mechanism, efficacy and safety. J Am Dent Assoc. 2000;131:589-596.

16. Hoang-Dao BT, Hoang-Tu H, Tran-Hung L, Camps J, Koubi G, About I. Evaluation of a natural resin-based new material (Shellac F) as a potential desensitizing agent. Dent Mater. 2008;24:1001-1007.

17. Gillam DG, Orchardson R. Advances in the treatment of root dentine sensitivity: mechanisms and treatment principles. Endod Topics. 2006; 13:13-33.

18. Kleinberg I, Kaufman HW, Wolff M. Measurement of tooth hypersensitivity and oral factors involved in its development. Arch Oral Biol. 1994;39(Suppl.):63S-71S.

19. Camps J, Salomon JP, Meerbeek BV, Tay F, Pashley D. Dentin deformation after scratching with clinically-relevant forces. Arch Oral Biol. 2003;48:527-534.

20. Boerio D, Hogrel JY, Creange A, Lefaucheur JP. Methods and clinical value of peripheral nerve refractory period measurement in man. Neurophysiol Clin. 2004;34:279-291.

21. Brännström M. A hydrodynamic mechanism in the transmission of pain producing stimuli through the dentine. In: Anderson JD, ed. Sensory mechanism in dentine. Oxford (UK): Pergammon Press, 1963:73-79.

22. Lukomsky EH. Fluorine therapy for exposed dentin and alveolar atrophy. J Dent Res. 1941;20:649-659.

23. Hoyt WH. Use of sodium fluoride for desensitizing dentin. JADA. 1943;30:1372-1376.

24. Thrash WJ, Jones DL, Dodds WJ. Effect of a fluoride solution on dentinal hypersensitivity. Am J Dent. 1992;5:299-302.

25. Camps J, About I, Van Meerbeek B, Franquin JC. Efficiency and cytotoxicity of resin-based desensitizing agents. Am J Dent. 2002;15:300-304.

26. Corona SA, Nascimento TN, Catirse AB, Lizarelli RF, Dinelli W, Palma-Dibb RG. Clinical evaluation of low-level laser therapy and fluoride varnish for treating cervical dentinal hypersensitivity. J Oral Rehabil. 2003;30:1183-1189.

27. Pamir T, Ozyazici M, Baloglu E, Onal B. The efficacy of three desensitizing agents in treatment of dentine hypersensitivity. J Clin Pharm Ther. 2005;30:73-76.

28. Ritter AV, de LDW, Miguez P, Caplan DJ, Swift EJ Jr. Treating cervical dentin hypersensitivity with fluoride varnish: a randomized clinical study. J Am Dent Assoc. 2006;137: 1013-1020. 
29. Addy M, West NX, Barlow A, Smith S. Dentine hypersensitivity: is there both stimulus and placebo responses in clinical trials? Int J Dent Hyg. 2007;5:53-59.

30. Doncaster CP, Davey AJ (eds). Analysis of variance and covariance. Cambridge (UK): Cambridge University Press; 2007.

31. Vieira AP, Lawrence HP, Limeback H, Sampaio FC, Grynpas M. A visual analog scale for measuring dental fluorosis severity. J Am Dent Assoc. 2005;136:895-901.

32. Gillam DG. Clinical trial designs for testing of products for dentine hypersensitivity - a review. J West Soc Periodontol Periodontal Abstr. 1997;45:37-46.

33. Ide M, Wilson RF, Ashley FP. The reproducibility of methods of assessment for cervical dentine hypersensitivity. J Clin Periodontol. 2001;28:16-22.

34. Orchardson R, Collins WJ. Thresholds of hypersensitive teeth to 2 forms of controlled stimulation. J Clin Periodontol. 1987; 14:68-73.

35. Kontturi-Nahri V, Narhi M. Testing sensitive dentine in man. Int Endod J. 1993;26:4.

36. Walline BW, Wagner JG, Marx DB, Reinhardt RA. Comparison of methods for measuring root and mucogingival sensitivity. Oral Surg Oral Med Oral Pathol Oral Radiol Endod. 2000;90:641-646.

37. Goodis HE, Tao L, Pashley DH. Evaporative water loss from human dentine in vitro. Arch Oral Biol. 1990;35:523-527.

38. Van Der Graat ER, Ten Bosch JJ. The uptake of water by freeze-dried human dentine sections. Arch Oral Biol. 1990;35:731-739.

39. Castillo JL, Milgrom P. Fluoride release from varnishes in two in vitro protocols. J Am Dent Assoc. 2004;135:1696-1699.

40. Gillam DG, Newman HN, Davies EH, Bulman JS, Troullos ES, Curro FA. Clinical evaluation of ferric oxalate in relieving dentine hypersensitivity. J Oral Rehabil. 2004;31:245-250.

41. de Assis Cde A, Antoniazzi RP, Zanatta FB, Rosing CK. Efficacy of Gluma Desensitizer on dentin hypersensitivity in periodontally treated patients. Braz Oral Res. 2006;20:252-256.

42. Addy M. Dentine hypersensitivity: new perspectives on an old problem. Int Dent J. 2002;52:367-375. 